\title{
Research on the Aesthetics of Current Chinese Three- dimensional Animation Based on Engine Animation
}

\author{
Yiran Fan \\ School of Digital Media \\ Chongqing College of Electronic Engineering \\ Chongqing, China
}

\author{
Yidong Liu \\ School of Digital Media \\ Chongqing College of Electronic Engineering \\ Chongqing, China
}

\begin{abstract}
Because "mechanical cinema" has affinity with three-dimensional animated movie, it should be called "engine animation". As a result, the game engines itself pursuits of the realistic and lifelike technology become as the transcript of the Chinese "engine animation" producers. In the context of postmodernity, the imitation of ending up being a "symbol" neglects the presence of historical culture, which makes "engine animation" and Chinese animated films become the replicas of symbol consumption. A large number of person with basic technical ability have been cultivated because of the pursuit of transcript, but we cannot blindly fall into such technological competition, and ignore the presence of historical culture and the audience's imagination of the animated films.
\end{abstract}

Keywords-engineer animation; animated film; aesthetics

\section{INTRODUCTION}

Machinima is formed by the combination of two English words Mechanical Cinema, translated as "mechanical cinema", or translated as "engine film", which is an image formed by the game engine without the help of other real people, real scene and other realistic elements and through CGI technology calculation. This kind of image should be called "animation" rather than "film", because the International Animation Film Association defines animation according to its charters: "In addition to the real-person and live-action shooting method, dynamic images should be created by the manipulation of various technologies.[1] Plus "engine animation" has an unusual "affinity" with three-dimensional animation in terms of production techniques - because the development of images developed and produced by game engines come from the earliest game interlude animations, with purpose to enrich the visual presentation, promote the story process and connect the context of the game. This kind of interlude animation and three-dimensional animation films all adopt offline rendering process, with homogeneity. As the 3D game engine matures, the frames of the game are getting closer to the image quality of the film, "so developers have gradually come to realize that they can use the game's own engine to render animation segments in the process of the game in real time." In this way, a great deal of manpower, material resources and time costs can be saved.[2] Therefore, the technology of "engine animation" is mature such as the vigorous development of realtime rendering in order to its realistic and lifelike image quality can catch up with the process presented by off-line rendering visual rendering and so on. Whether A Boy and His
Kite [3], an animated short film calculated by EPIC's Unreal 4 in 2015, Adam [4], an animated short film based on Unity3D game engine calculation in 2016, or the high-fidelity real-time digital role 'Siren' that makes a stage pose at GDC in 2018 produced by Chinese actress Jiang Bingjie's image, the technology of "engine animation" are recognized in the above three examples. The fascination with the display effect under the standard of digital technology, which leads the world, influences the aesthetic concept of Chinese three-dimensional animators.

\section{A Kind of Magic - Aesthetic Thinking On IMAge PURSUIT OF "NATURAL REALITY"}

Before the analysis of the impact of the digital technological innovation of "Game engine" on the visual aesthetics of Chinese three-dimensional animated films. Perhaps from Andre Bazin's expression about "realism" we can find the original impulses for technological innovation generated by the game engine's fascination with "natural reality. That is, the obsession with "reality" may be due to the "mummy plot" of the human subconscious. Bazin believes that the invention of the painting technique of "perspective method" in the 15 th century enables painters to create threedimensional fantasies with a system of initial mechanical characteristics, and that "once this demand for pursuing fantasy is met, and it becomes increasingly stronger so that gradually engulfs plastic arts."[5] Such pursuit of realistic fantasy constantly influences painting from the inside, and Bazin believes that this is a kind of "pure psychological demand, which does not belong to the category of aesthetics, and can only be found from the psychology of pursuing magic."[6] Such primitive force has been explored with another layer of meaning in Norman Bryson's Vision and Painting: Logic of Gaze, that is, the re-representation of "natural reality" comes from the competition among painters [7] Gabriela. F. Oriol Giralt quoted Bryson's views on the competition, and he said that "the digital work flow was actually a platform that artists can compete with one another to pursue the transcendence of realism in this process created by their predecessors."[8] However, the game engine seems to act as such a role. The developers' pursuits of lifelike effect standard seem to be becoming a transcended facsimile of the participants behind them, and such transcendent will causes creators to ignore the investigation of historical presence, and then obliterates the style existence in the creation and causes 
the picture to be close to "the standardization". Such real competition for "beyond reality" itself can be understood as a microscopic interpretation of "hyper-reality" in Baudrillard's vision. This kind of "reality" is more like a code, which affects the creation of animation arts.

A more severe expression is that the world's outstanding digital artists who make digital fantasy through game engines seem to be becoming an ideal contender for Chinese game animators, and we may abandon the presence of history. For example, we may ignore the thought on the traditional Chinese cultural heritage and the current social context and unlimited fall into the competition that has a "realistic" color, but in fact it is just an one of abstract symbolic image, which may undoubtedly make the Chinese producers of "engine animation" lost the investigation of the presence of history and fall into a quagmire of a kind of imaginary "symbol" imitation. This is what Jean Pierre Orgone criticized. For students who graduate from the film school in recent years, they desperately show off their skills to the film industry system that has become a perfect competition" [9].

\section{SymbolizATION TREND OF ENGINE ANIMATION}

$\mathrm{Wu}$ Guanping believes that the aesthetic form of Chinese film in the recent decade can be divided into two types of aesthetics: "one is Hollywood-style Chinese high-concept" film and the other is the local "interesting community films on the Internet". Based on "engine" concept, a large number of films that spread on the Internet, such as "The Bad Man" series produced by Northen Digital Technology with its selfdeveloped mandala system, The legend of Qin, Tian Xing Jiu Ge and Tian Yu produced by Sparkly Key Animation Studio, and Di Ling Qu produced by Zhuohua Network Technology with Unreal 4 engine belong to the category of "interesting community on the Internet". Throughout the animation style of these "interesting community on the Interne", they all show a kind of highly "symbolized" and "standardized" purport: (a) realistic and lifelike expression of the space scene; (b) simple and fashionable character modeling; (c) emulational character action design; (d) simple character expression shaping. These purports are related to the technical indicators of the game engine and the acceptance level of the current mainstream game character modeling styles in the market. The formation may be attributed to two factors, firstly as David Cheney said (cultural economic activities) that there has been a circulation form from material handicrafts trade to the symbolic crafts trade... In a more general sense, all kinds of goods, activities and environments are treated as reappearances of concentrated meaning and association to accept valuation and conduct deal". [10] Animations developed through the principles of game engine are bound to be subject to the mechanism factors. For example, the perfect copy of a set of closed "engine animation" standards, and the products (animations) created by it will certainly please the aesthetic perceptions and consumer beliefs of a certain group of target audience because the narrative routines of interesting community on the Interne" and visual conventions have not yet formed a "contract" with the target audience of the greatest common divisor"[11], which eventually form a highly concise superficial symbol. Secondly, as mentioned above, the willingness of the contest among producers may accelerate and solidify the process of symbolization, which is not just an imitation of the game engine's "realistic" standards (so you can see more and more real texture representation, physical dynamics calculations and special effects, which are also sources of technological innovation for game engines), and more is the competition from peers (occupying the market).

\section{Aesthetic Dilemma of ThreE-DimEnsional ANIMATION}

The influence of the "symbol" imitation of "engine animation" on the creation of Chinese three-dimensional animation films is not direct but circuitous, which can be found proofs from the industrial pattern and the text itself. First of all, Xuanji technology and Norson Digital that are the first to create the "engine animation" has begun to shift to the field of animated films, they must follow its existing aesthetic purports to upgrade the screen so that works in the style of creation lack of innovation. Secondly, in the "2012 Employment Report of Chinese College Student" written by the "Mikesi" Research Institute, the animation major is highlighted as "red card", [12] which marks the embarrassing situation of being unemployed after graduation for the animation talents, but actually due to the market size and economic benefits of the game and animation industries in different periods have led to the movement of talents between the two industries. For example, more students from animation major have transferred to the game industry after graduation and those who have transferred from the game industry to the animation industry because the economic benefits of the animation industry. [13] Finally, cross-industry personnel mobility may form an emotional field of aesthetics and dominate the will of industry personnel, that is, the imitation of "symbols" by technical producers. But the way of such "symbol" imitation from a single specification of the technical indicators of the game engine that is a digital platform further forms a pluralistic form. That is, making the modeling, rendering, performance in "Disney", "Pixar" and "Dream Factory", and the lens language and narrative principles as a competition object of self-recognition and going all out to copy and transcend. Just like the Light Chaser Animation Studios Wang Wei says that "we may have the opportunity to directly cross the next generation when the new thing comes out such as lighting rendering. As for lighting rendering, we don't have to use the old rendering method and we can directly enter the rendering of the new real physical lighting... We hope that when we say the second and third films of our work that is not simply similar to Disney, we hope that we can do better." [14]

Therefore, as for the Rock Tibetan Mastiff, the Adventures of Ah Tang, the Legend of Tofu, the Cat and the Peach Garden and Mamma Mi Ya, they are all not perfect in the choice of traditional Chinese cultural symbols, the narrative content on the contemplation of contemporary life and the market return. So the convergence of visual presentation style and performance characteristics also makes the main body of Chinese animated films to be self-effacement in this imitation of "symbol". 


\section{CONCLUSION}

If the painting competition makes the painting "realism" separate from the investigation of presence of the real social history, causes the limitation of style and the transmission mode of a kind of perceptionism. As for the creation of animated film in the context of post-modern culture, due to the complexity of the industry is subject to the symbolization trend caused by "engine animation", the creation of threedimensional animation is affected. It seems to be unconscious to fall into a creative shackle in the creative process because such competition ignores the investigation of the presence of historical culture and lacks the sense of innovation as required, which won't not only achieve the Chinese audience's imagination of Chinese animated films, but also highlight the local cultural self-confidence and cultural consciousness. Wang Yichuan believes that "the self-entertainment era of the current Chinese film industry needs the secret worry that needs to introspect deeply. Perhaps it is exactly the strong rise of commercial aesthetic principles and the consumerist belief behind which has already become the guide to the moviewatching behavior of the netizens and even the guide to the conduct of daily life as a whole.[15]" The creation of symbolic form should not be totally negated, but should be dialectically understood. Firstly, the "standardization" of images enables animation as a commodity to have a more predictable benefit prediction, that is, it conforms to the consumer beliefs brought by the general aesthetic perception of a certain group. Secondly, what "competition" brings is the technical reserve of Chinese animated films, and we should not follow the industrial aesthetics concepts brought by the single technical standard when we "compete". At the same time we should expand our own talented persons with innovative skills, and we do not degenerate the creation into a transcendental and non-stylistic copy.

\section{REFERENCES}

[1] Zhu Jian. The Study of Chinese Animation Art, Nanjing: Southeast University Press,2012 Version, Page.1-2 . (in Chinese)

[2] Wang Daiming. Engine Film, the Integration of Video Games and Movies, Movie Art, No. 3, 2007, Page.123-124. (in Chinese)

[3] A boy and His Kite is an animated short film that is calculated by EPIC's Unreal 4 game engine in real-time rendering, which is presented at the 2015 Computer Graphics and Images Conference. Its images springs to life with dynamic direct and indirect illumination, cinematic depth of field and high-quality motion blur and procedurally placed trees and foliage and real-time rendering. Please refer to https://www.unrealengine.com/en-US/blog/siggraph-unreal-kite-bestreal-time-graphics-interactivity-award. (in Chinese)

[4] "Adam" is a real-time rendered short film produced by using the Unity engine and wins the 2017 Webby Award. Adam is originally released in 2016 to demonstrate and test the graphic rendering capabilities of the Unity engine. Its developers use real-time regional lighting and highfidelity physics simulation tools CaronetFX and some custom tools such as volume fog, dynamic blur to meet and extend the real-time rendering realism.Please refer to https://unity3d.com/cn/pages/adam. (in Chinese)

[5] Li Hengji, Yang Yuanying. Selected Works of Foreign Film Theory, Beijing: Sanlian Bookstore, 2006 Version, Page. 281-284. (in Chinese)

[6] Li Hengji, Yang Yuanying. Selected Works of Foreign Film Theory, Beijing: Sanlian Bookstore, 2006 Version, Page. 281-284. (in Chinese)

[7] Written by Norman Bryson and Translated by Guo Yang etc. Vision and Painting: Logic of Gaze, Zhejiang: Sanlian Bookstore, Zhejiang Photography Publishing House, 2004 Version, Page 9-12. (in Chinese)
[8] [US]Gabriel. F. Oriol Giralt. The Interchangeability of Visual Effects and Physical Photography and Its Rewriting of The Meaning of Realism, World Movie, No. 1, 2018, Page. 11.

[9] [US] Jean Pierre Orgone. On The Fate of Cinema in The Early 21st Century, World Cinema, No. 1, 2004, Page. 10.

[10] Wu Guanping. Aesthetic Changes in Contemporary Chinese Cinema, Contemporary Film, No. 2, 2018, Page. 114-115. (in Chinese)

[11] Wu Guanping. Aesthetic Changes in Contemporary Chinese Cinema, Contemporary Film, No. 2, 2018, Page. 114-115. (in Chinese)

[12] Why Animation Major "Red Card" Strobe, NetEase News. August 11, 2012, http://news.163.com/12/0811/08/88K4RKA700014AED.html. (in Chinese)

[13] San Wen Entertainment Animation Talent report No. 1: The Trend of Salary Rise, Game Movie Talents to Animation, 2D Animation Practitioners to 3D ", Sanwen Entertainment. February 15, 2017, http://www.3wyu.com/11051.html. (in Chinese)

[14] "Chasing the Light and These Three Years of Wangwei: Sharpening the Sword, Practicing Qi, Gathering three Internal Forces, LightChaser Animation, March 13, 2013, http://www.zhuiguang.com/?p=877. (in Chinese)

[15] Wang Yichuan. Artistic Aesthetics Poverty and Commercial Aesthetics Rich and their Harmony - the Aesthetic Landscape of domestic Films of 2015", Contemporary Film, No. 3, 2016, page. 24. (in Chinese) 